\title{
Minimally invasive staging of esophageal cancer
}

\author{
Kunal Mehta, Valentino Bianco, Omar Awais, James D. Luketich, Arjun Pennathur \\ Department of Cardiothoracic Surgery, University of Pittsburgh School of Medicine and University of Pittsburgh Medical Center, Pittsburgh, PA, USA \\ Correspondence to: Dr. Arjun Pennathur, MD, FACS. Department of Cardiothoracic Surgery, University of Pittsburgh Medical Center, 200 Lothrop \\ Street, Suite C-800, Pittsburgh, PA 15213, USA. Email: pennathura@upmc.edu.
}

\begin{abstract}
Esophageal cancer is one of the most common malignancies in the world today and the sixth-leading cause of cancer-related mortality. Accurate preoperative staging of esophageal cancer is imperative to the selection of appropriate treatments. Patients with esophageal carcinomas typically undergo a multimodality staging process including noninvasive imaging techniques, such as computed tomography (CT) and positron emission tomography (PET), as well as endoscopic ultrasound (EUS), which is slightly more invasive. Minimally invasive surgical staging, with laparoscopy, occasionally in combination with video-assisted thoracoscopy, is used in the staging process at select institutions and has been shown to be more accurate than noninvasive staging modalities. Two major advantages of minimally invasive surgical staging over conventional techniques are the improved assessment of locoregional disease and enhanced identification of distant metastases. These advantages decrease the likelihood that the patient will undergo a nontherapeutic laparotomy. Currently, no clear consensus exists regarding which patients with esophageal cancer would benefit most from the addition of minimally invasive surgical staging. We have, however, found that minimally invasive surgical staging with laparoscopy is particularly valuable in detection of occult distant metastases. In this article, we summarize the staging modalities for esophageal cancer including minimally invasive surgical staging.
\end{abstract}

Keywords: Esophageal cancer; laparoscopy; minimally invasive surgery (MIS); staging; thoracoscopy

Submitted Jan 19, 2016. Accepted for publication Mar 21, 2017.

doi: $10.21037 /$ acs.2017.03.18

View this article at: http://dx.doi.org/10.21037/acs.2017.03.18

\section{Introduction}

\section{Esophageal cancer}

Esophageal carcinoma is currently the sixth-leading cause of cancer-related mortality and the eighth most common cancer worldwide. There are more than 450,000 individuals afflicted with esophageal cancer today. There has been an epidemiological shift in the histology of esophageal carcinoma, with a rising incidence of adenocarcinoma as compared with squamous cell carcinoma in the Western population (1). It is estimated that in 2017 the United States will see 16,940 new cases of esophageal cancer and 15,690 deaths from esophageal cancer (2). The prognosis for patients with esophageal cancer remains poor with 5 -year survival rates estimated at $15-25 \%(1,3,4)$. These poor outcomes are due in part to the typical presentation of the disease with diagnosis occurring at late (metastatic) stages and the propensity for even superficial tumors to metastasize $(1,3,5)$. Survival is improved when esophageal cancer is detected at an earlier stage (5). Once the diagnosis of esophageal cancer is made, accurate staging is essential to ensure that the correct treatment protocols are applied. Tumor stage, estimated prognosis, and the patient's physiological status are assessed together to develop the proper treatment plan for each individual.

\section{Esophageal cancer staging}

Esophageal cancer is currently staged according to the TNM (tumor, node, metastasis) staging system. This staging system classifies lesions based upon depth of tumor invasion ( $\mathrm{T}$ stage), regional lymph node status ( $\mathrm{N}$ stage) and the presence or absence of distant metastasis ( $M$ stage) (Figure 1). The TNM system was first introduced by the 


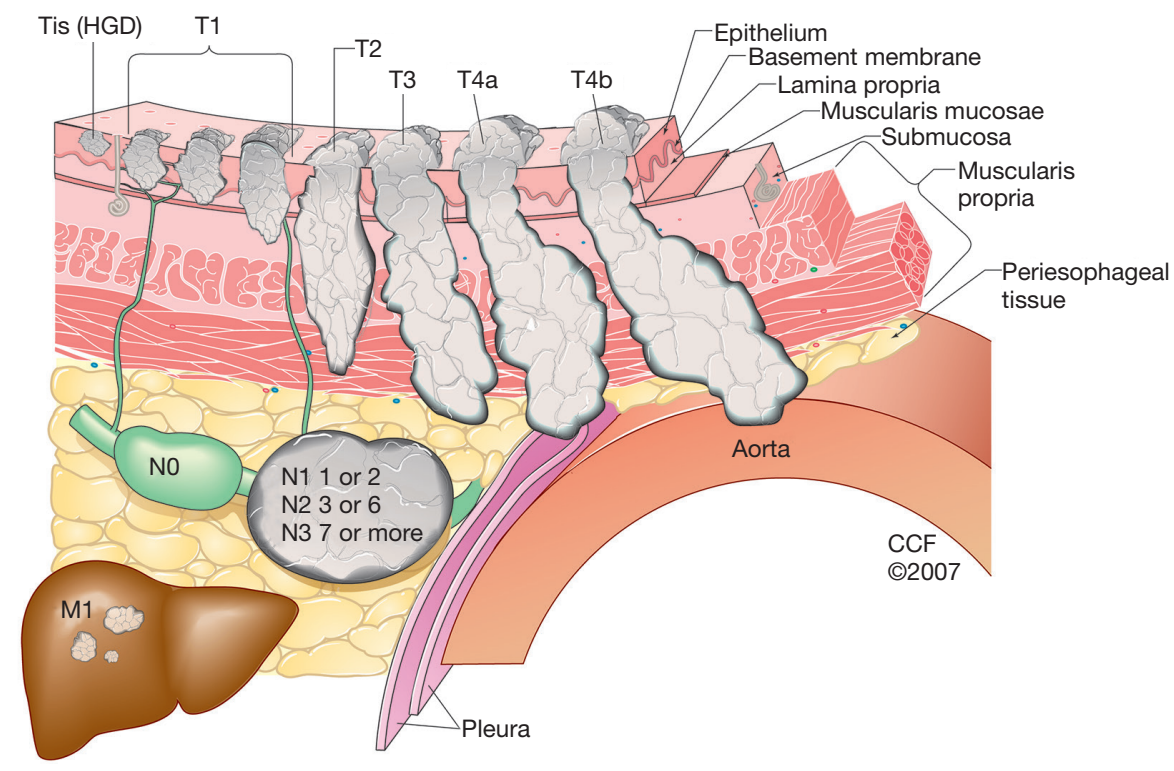

Figure 1 Features used to stage esophageal carcinoma according to the TNM classification system. Reprinted with permission, Cleveland Clinic Center for Medical Art \& Photography @2007-2017. All Rights Reserved.

International Union Against Cancer (UICC) in 1968 and has undergone multiple revisions since that time (4). In 2010, the American Joint Committee on Cancer (AJCC) released the seventh edition staging system for esophageal cancer (6). Notable modifications to previous staging classifications made in this most recent edition include the classification of $\mathrm{T} 4$ lesions as resectable (T4a) or unresectable (T4b), the stratification of $\mathrm{N}$ stage by number of nodes involved, and the stratification of stage according to tumor histology, degree of differentiation (grade), and location (1,7-9) (Table 1). In the future, refinements in molecular staging of esophageal cancer with analysis of gene expression profiles may also play a role in risk stratification and in the treatment algorithm of esophageal cancer (10).

\section{Clinical presentation and evaluation}

A multimodality approach is necessary to determine the stage of an esophageal tumor. Esophageal cancer patients typically present with clinical symptoms such as dysphagia and weight loss. Evaluation begins will a full history and physical examination along with a barium esophagogram and endoscopy. The barium esophagogram typically shows an esophageal stricture where a lesion is present. Endoscopy is essential for a definitive diagnosis; it reveals the tumor location and length and permits biopsy for pathological examination. In addition, bronchoscopy is performed in patients with proximal or midesophageal lesions to exclude airway involvement. Patients routinely undergo a computed tomography (CT) scan of the chest and upper abdomen, which is useful in assessing local spread of disease and distant metastasis. Positron emission tomography (PET) is increasingly being used in the evaluation of distant metastasis. Endoscopic ultrasound (EUS) is performed to assess $T$ stage by providing detailed information about the esophageal wall. Additionally, EUS is useful for evaluation of $\mathrm{N}$ status. Finally, at some institutions, minimally invasive surgical (MIS) staging with laparoscopy, and occasionally with thoracoscopy is performed in select patients. In this article, we review the literature regarding non-invasive and minimally invasive staging of esophageal carcinoma and comment on the accuracy and utility of MIS techniques as compared with conventional staging methods.

\section{Staging modalities}

\section{Noninvasive imaging}

Owing to its relatively low cost and widespread availability, CT imaging of the chest and abdomen is frequently obtained in esophageal cancer patients to evaluate for both locally advanced disease and distant metastasis. CT imaging is helpful in depicting the tumor growth and the loss of 
Table 1 Stage classification of esophageal carcinoma according to the 2010 AJCC 7th Edition staging system

\begin{tabular}{|c|c|c|c|c|c|}
\hline Stage & $\mathrm{T}$ & $\mathrm{N}$ & M & $\mathrm{G}$ & Location \\
\hline \multicolumn{6}{|c|}{ Esophageal adenocarcinoma } \\
\hline 0 & Tis (HGD) & 0 & 0 & 1 & NA \\
\hline IA & 1 & 0 & 0 & $1-2$ & NA \\
\hline \multirow[t]{2}{*}{ IB } & 1 & 0 & 0 & 3 & NA \\
\hline & 2 & 0 & 0 & $1-2$ & NA \\
\hline IIA & 2 & 0 & 0 & 3 & NA \\
\hline \multirow[t]{2}{*}{ IIB } & 3 & 0 & 0 & Any & NA \\
\hline & $1-2$ & 1 & 0 & Any & NA \\
\hline \multirow[t]{3}{*}{ IIIA } & $1-2$ & 2 & 0 & Any & NA \\
\hline & 3 & 1 & 0 & Any & NA \\
\hline & $4 a$ & 0 & 0 & Any & NA \\
\hline IIIB & 3 & 2 & 0 & Any & NA \\
\hline \multirow[t]{3}{*}{ IIIC } & $4 a$ & $1-2$ & 0 & Any & NA \\
\hline & $4 b$ & Any & 0 & Any & NA \\
\hline & Any & 3 & 0 & Any & NA \\
\hline IV & Any & Any & 1 & Any & NA \\
\hline
\end{tabular}

Esophageal squamous cell carcinoma

$\begin{array}{llllll}0 & \text { Tis (HGD) } & 0 & 0 & 1 & \text { Any } \\ \text { IA } & 1 & 0 & 0 & 1 & \text { Any } \\ \text { IB } & 1 & 0 & 0 & 2-3 & \text { Any } \\ & 2-3 & 0 & 0 & 1 & \text { Lower } \\ \text { IIA } & 2-3 & 0 & 0 & 1 & \text { Upper, middle } \\ & 2-3 & 0 & 0 & 2-3 & \text { Lower } \\ \text { IIB } & 2-3 & 0 & 0 & 2-3 & \text { Upper, middle } \\ & 1-2 & 1 & 0 & \text { Any } & \text { Any } \\ \text { IIIA } & 1-2 & 2 & 0 & \text { Any } & \text { Any } \\ & 3 & 1 & 0 & \text { Any } & \text { Any } \\ & 4 a & 0 & 0 & \text { Any } & \text { Any } \\ \text { IIIB } & 3 & 2 & 0 & \text { Any } & \text { Any } \\ \text { IIIC } & 4 a & 1-2 & 0 & \text { Any } & \text { Any } \\ & 4 b & \text { Any } & 0 & \text { Any } & \text { Any } \\ & \text { Any } & 3 & 0 & \text { Any } & \text { Any } \\ \text { IV } & \text { Any } & \text { Any } & 1 & \text { Any } & \text { Any }\end{array}$

T, tumor classification; N, lymph node status; M, metastasis; G, histologic grade; Tis, tumor in situ; HGD, high-grade dysplasia; NA, not applicable. Modified from (9); used with permission. fat planes between the esophagus and surrounding tissues, which is suggestive of local disease and inflammation. While CT imaging has limited utility in the assessment of depth of tumor invasion through the esophageal wall due to its resolution, it potentially increases the accuracy of the evaluation of the primary tumor and adjacent organ involvement (e.g., aortic involvement). The highest utility of CT imaging is in the identification of distant metastases to organs such as the lung or liver $(8,11,12)$.

Computed tomography can also identify enlarged lymph nodes that raise suspicion of tumor involvement (11). The results of several studies have raised concerns over the accuracy of CT imaging in identifying small nodal metastases. In 2000, Luketich and colleagues noted that the sensitivity and specificity of CT scan of the chest and abdomen for lymph node metastases were $33 \%$ and $88 \%$, respectively. Moreover, CT imaging proved to be inaccurate in more than $40 \%$ of patients as compared with minimally invasive staging (13).

PET imaging is increasingly being applied in the staging of esophageal cancer. PET, like CT, is limited in the assessment of $\mathrm{T}$ stage, and its primary benefit is in the detection of distant metastatic disease $(1,14,15)$. In fact, studies have shown that PET imaging can identify distant metastases in patients with a negative CT scan in 10-20\% of cases (13). The reported accuracy of PET in detection of nodal metastases varies, ranging from $27 \%$ to $90 \%(1,15)$. The combination of PET and CT is superior to PET alone in the assessment of nodal staging, but its reliability for assessing locoregional lymph node involvement is questioned, particularly when the lymph node is in close proximity to the primary tumor. False-positive nodes in PET imaging have also been noted in patients with airway inflammation from chronic lung diseases or previous tuberculosis (12).

\section{Endoscopic ultrasound}

Since its introduction into clinical practice in the 1980s, EUS has rapidly grown into a reliable technique for assessing lesions of the GI tract. EUS visualization can display five distinct layers of the esophageal wall (the echo/ superficial mucosa boundary, the mucosa, the submucosa, the muscularis propria, and the adventitia) and represents an important advance in esophageal cancer staging. The reported sensitivity of EUS in the assessment of T-stage is $85-90 \%$, and the reported accuracy is $70-80 \%(16,17)$. EUS also is useful in detecting regional lymphadenopathy and sampling suspicious nodes with fine-needle aspiration (FNA) 


\begin{tabular}{|c|c|c|c|c|c|}
\hline \multirow{2}{*}{ Studies } & \multirow{2}{*}{$\begin{array}{l}\text { Number of } \\
\text { patients }\end{array}$} & \multirow{2}{*}{ Histology } & \multicolumn{3}{|l|}{ EUS $^{a}$} \\
\hline & & & Accuracy (\%) & Sensitivity (\%) & Specificity (\%) \\
\hline Luketich et al., 1997 (18) & 26 & 24 (92\%) AC; 2 (8\%) SCC & 65 & 65 & 66 \\
\hline Luketich et al., 2000 (13) & 53 & 46 (87\%) AC; 7 (13\%) SCC & 62 & 62.5 & 60 \\
\hline
\end{tabular}

AC, adenocarcinoma; EUS, endoscopic ultrasound; SCC, squamous cell carcinoma. ${ }^{\text {a }}$, Nodal status as determined by laparoscopy and/ or thoracoscopy was used to assess accuracy, sensitivity and specificity of EUS; ${ }^{b}, 90 \%$ accuracy for nodal staging when used for FNA; $72 \%$ overall staging accuracy.

(Table 2). In a study by Kaushik and colleagues, EUS with FNA had an accuracy of $72 \%$ for overall staging and $90 \%$ for nodal staging $(1,19)$.

Nonetheless, EUS has its limitations, and several controversies remain regarding the role of EUS in the clinical staging of esophageal cancer. Because there has been a recent surge in the use of endoluminal therapies, such as endoscopic mucosal resection (EMR), radiofrequency ablation, and photodynamic therapy, for treatment of superficial tumors limited to the muscularis mucosa (TisT1a), it has become increasingly important to accurately stage early-stage lesions. EUS has poor accuracy in the assessment of superficial lesions (20,21). A study by Bianco and colleagues from the University of Pittsburgh demonstrated that EUS accurately staged just $39 \%$ of T1a lesions and 70\% of T1b lesions (20). Similarly, Bergeron and colleagues at the University of Michigan found that only $39 \%$ of $\mathrm{T} 1 \mathrm{a}$ lesions and $51 \%$ of $\mathrm{T} 1 \mathrm{~b}$ lesions were correctly staged using EUS. Moreover, in Bergeron's study, lesions thought to be cT1aN0 by EUS turned out to be pN1 or higher in $15 \%$ of patients; endoluminal therapy would undertreat these patients $(16,21)$. Luketich and colleagues determined that even when EUS and CT were combined, staging inaccuracies were present in up to $32 \%$ of patients. In addition, there is additional concern that the accuracy of EUS may be further reduced in patients with advanced disease who receive neoadjuvant chemotherapy or radiotherapy (13).

\section{Endoscopic mucosal resection}

Endoscopic therapies for esophageal cancer are widely utilized in the treatment and palliation of advanced or inoperable tumors. Recently, there has been increased demand for less invasive techniques that promise reduced morbidity or mortality for patients with early-stage esophageal cancers. As such, EMR is emerging as both a diagnostic and therapeutic modality in highly selected patients with superficial esophageal lesions.

As discussed above, no imaging technique, including EUS, is accurate in distinguishing mucosal (T1a) lesions from submucosal (T1b) lesions. EMR may be useful in staging superficial esophageal lesions due to this capability. EMR excises a disc of tissue from the esophageal wall with a resection plane at the interface of the submucosa and muscularis propria. Histopathological assessment of EMR specimens allows for the accurate identification of submucosal invasion, which is associated with high rates of lymph node involvement and warrants esophagectomy (22). In addition, patients with intramucosal cancer, particularly with adverse factors such as poor differentiation or angiolymphatic invasion, are treated with esophagectomy (5). A small study by Maish and DeMeester stated that EMR was accurate in determining depth of tumor invasion in $100 \%$ of the patients and completely excised the tumor in $86 \%$ of these cases. They recommend EMR as an adjunct to EUS in the staging of early esophageal lesions in all cases when the ultimate therapy does not include a lymphadenectomy $(4,23)$.

\section{Minimally invasive surgical staging}

Minimally invasive staging in esophageal cancer includes the techniques of laparoscopy and thoracoscopy for evaluation of esophageal tumors. While not widely practiced, minimally invasive staging can have clear benefits for select individuals. The Society of Thoracic Surgeons recommends staging laparoscopy for locally advanced (T3/ T4) adenocarcinoma of the gastroesophageal junction (GEJ) that infiltrates the gastric cardia (24). The National 
Comprehensive Cancer Network considers laparoscopy an optional part of the staging process in patients with GEJ lesions without evidence of metastatic disease (25). Two major advantages of MIS staging are the potential avoidance of a nontherapeutic laparotomy (and its associated mortality and morbidity) due to enhanced detection of distant metastases and the identification of more patients who might benefit from neoadjuvant therapy due to improved detection of locally advanced disease $(1,24,26,27)$.

\section{Surgical techniques for minimally invasive staging}

Laparoscopic staging is typically performed under general anesthesia with the patient in the supine position. All quadrants of the peritoneal cavity and the liver surface are inspected for suspicious metastatic deposits with samples of grossly abnormal areas sent for pathological examination. Intraoperative ultrasonography of the liver can also be performed using a flexible-tip laparoscopic ultrasonography probe. Lymph nodes of the celiac axis may be examined by dissecting through the lesser omentum to expose the left gastric vessels, evaluating for bulky and fixed lymphadenopathy and assessing resectability $(11,27,28)$.

Thoracoscopic staging is generally carried out using video-assisted thoracoscopy (VATS) and single-lung ventilation with the patient in the lateral decubitus position. This approach may be utilized selectively to exclude possible metastases (e.g. when there is concern for metastasis to the lung or pleura) or possible involvement of adjacent organs, such as the aorta (11,27-29).

\section{Benefits of minimally invasive staging}

One of the key advantages of minimally invasive staging using laparoscopic and/or VATS techniques (used selectively) is the improved assessment of locoregional disease. Several studies have established the superior sensitivity of minimally invasive staging in identifying small foci of metastatic disease affecting lymph nodes (19,30,31). Luketich and colleagues carried out a prospective study to assess the accuracy of EUS and minimally invasive techniques in identifying lymph node metastases in patients with esophageal cancer. The sensitivity and specificity of EUS for identifying nodal disease were $65 \%$ and $66 \%$, respectively. The sensitivity of EUS for identifying nodal disease decreased to $44 \%$ for nodal metastases less than $1 \mathrm{~cm}$ in diameter. MIS staging revealed N1 disease in 6 of 8 patients previously thought to have N0 disease (19). Moreover, while endoscopic ultrasonography was unable to detect distant metastases in any patient, MIS staging identified liver metastases in 4 of $26(15 \%)$ patients. Minimally invasive staging was, therefore, shown to increase the accuracy of staging lymph node metastases with the added advantage of detection of occult metastases. Similarly, Kaushik and colleagues noted that even though EUS-FNA had a $90 \%$ accuracy for nodal staging in 47 patients who underwent both EUS and laparoscopic staging, EUS-FNA only had a $72 \%$ accuracy in overall staging when compared with MIS staging. The majority of staging differences were due to the detection of distant metastases during MIS staging, providing the rationale for the inclusion of MIS in staging algorithms for esophageal cancer (18). A study by Chang and colleagues found that MIS staging had an overall sensitivity of $78 \%$ in the detection of nodal metastases, as compared with $11 \%$ for EUS alone and $55 \%$ for CT (31).

To date, there has been one prospective multicenter, cooperative-group trial in the United States (CALGB 9380) investigating the feasibility of minimally invasive staging in esophageal cancer. CALGB 9380 was a multiinstitutional National Cancer Institute-funded phase II trial that included 134 patients. The primary objective of the study was to evaluate the feasibility of MIS as a staging modality. Secondary goals included assessing the accuracy of MIS staging and benefit when added to clinical staging. Minimally invasive staging was technically feasible in $73 \%$ of patients with no mortality or major complications. Positive nodal disease was found in $56 \%$ of patients, and MIS staging doubled the number of positive nodes identified by conventional, noninvasive imaging (30).

There is also data supporting the improved efficacy of MIS staging in detecting distant metastases, when compared with conventional imaging (Table 3). MIS staging has a role in patients with potentially resectable esophageal cancer without distant metastases detected by conventional imaging. For these patients, laparoscopy can be used to identify small liver surface and peritoneal metastases while thoracoscopy can assess invasion of local structures, such as the aorta or pleura $(26,28,32-35)$. In an interesting study, Luketich and colleagues prospectively studied the staging accuracy of PET and MIS staging in patients with potentially resectable disease. They evaluated 100 consecutive PET scans in 91 patients with potentially resectable carcinoma of the esophagus to compare staging accuracy with conventional imaging methods. They found PET imaging to be significantly more accurate than CT in the identification of distant metastases (accuracy $84 \%$ vs. 63\%, $\mathrm{P}<0.01)$. Nonetheless, PET was still only $69 \%$ sensitive in identifying distant metastases as compared with MIS staging 


\begin{tabular}{|c|c|c|c|c|c|c|c|c|}
\hline \multirow[b]{2}{*}{ Studies } & \multirow{2}{*}{$\begin{array}{l}\text { Number } \\
\text { of } \\
\text { patients }\end{array}$} & \multirow[b]{2}{*}{ Histology } & \multicolumn{3}{|l|}{ MIS } & \multicolumn{3}{|c|}{ Conventional imaging } \\
\hline & & & $\begin{array}{l}\text { Accuracy } \\
(\%)\end{array}$ & $\begin{array}{l}\text { Sensitivity } \\
(\%)\end{array}$ & $\begin{array}{l}\text { Specificity } \\
(\%)\end{array}$ & $\begin{array}{l}\text { Accuracy } \\
(\%)\end{array}$ & $\begin{array}{l}\text { Sensitivity } \\
(\%)\end{array}$ & $\begin{array}{l}\text { Specificity } \\
(\%)\end{array}$ \\
\hline de Graaf et al., 2007 (26) & 416 & 375 (90\%) AC; 33 (8\%) SCC & -- & 88 & 100 & $67^{\mathrm{b}}$ & $66^{\mathrm{b}}$ & -- \\
\hline Nguyen et al., 2001 (28) & 33 & 24 (73\%) AC; 9 (27\%) SCC & 97 & 96 & 100 & $61^{\mathrm{c}}$ & $100^{c}$ & $91^{\mathrm{c}}$ \\
\hline Romijn et al., 1998 (32) & 40 & 25 (60\%) AC; 15 (40\%) SCC & 95 & 70 & 100 & -- & -- & -- \\
\hline \multicolumn{9}{|c|}{$\begin{array}{l}\text { a , Unresectable disease was due to distant metastasis, locally advanced disease, or extensive lymph node involvement; }{ }^{b} \text {, conventional } \\
\text { imaging with CT and EUS; }{ }^{~} \text {, conventional imaging with CT only; }{ }^{d} \text {, distant metastatic disease was determined by MIS or clinical correlation } \\
\text { and used to assess accuracy, sensitivity, and specificity of PET. All other studies compared MIS staging and conventional imaging } \\
\text { against the gold standard of surgical resection/exploration; }{ }^{\text {, }} \text {, conventional imaging by CT followed by PET. AC, adenocarcinoma; NR, not } \\
\text { reported; SCC, squamous cell carcinoma. }\end{array}$} \\
\hline
\end{tabular}

(Figure 2) (15). In this study, there were 18 occult metastatic lesions missed by PET scan and detected by MIS staging. All these were less than $1 \mathrm{~cm}$ in diameter. The predominant sites of occult metastases included the liver, pleura, and lung. These findings suggest that minimally invasive staging may be useful in the detection on unsuspected distant metastases after PET scanning.

Similarly in a series of 33 patients, Nguyen and colleagues found that MIS staging accurately determined resectability in $97 \%$ of patients, while conventional imaging techniques accurately determined resectability in just $61 \%$ of patients. The use of MIS staging avoided an unnecessary laparotomy in 10 of their patients (28). In this study, laparoscopy was routinely performed, and VATS was selectively performed when there were equivocal findings. One patient in their series underwent VATS resection of a suspicious lung nodule, which turned out to be benign and patient went on for definitive resection. The investigators noted that there was a single patient in this series with a false-negative staging procedure, and no thoracoscopy was performed. This patient had unsuspected pleural metastases, which could have been detected if thoracoscopy had been performed. The largest investigation into this area occurred in 2007 when de Graaf and colleagues retrospectively reviewed the records of 416 patients with esophageal cancer referred to their center over a 7 -year period and found that MIS staging had a sensitivity of $88 \%$ and specificity of $100 \%$ for identifying operable disease. An unnecessary laparotomy was avoided in 63 patients $(6.6 \%)$ with peritoneal or liver metastases (26).

MIS staging alters stage classification and the associated treatment plan in $30-40 \%$ of patients $(13,28)$. Therefore, it may be appropriate to consider the use of MIS staging in patients with potentially resectable disease as determined by conventional imaging to increase the appropriate administration of multimodality therapy including neoadjuvant therapy and surgical resection and avoid potential cases of nontherapeutic laparotomy. Given the clinically silent nature of esophageal cancer, patients frequently present with locally advanced disease, which is best treated with a multimodality approach. Patients who are thought to have N0 disease following conventional imaging may typically proceed to esophagectomy. As described above however, MIS staging results in upstaging in a considerable percentage of patients when compared with nodal staging by conventional techniques. Therefore, MIS staging may identify patients with nodal metastases, and stratify these patients into a more appropriate treatment algorithm using a multimodality therapy, which is particularly important in due to the propensity for early nodal metastasis $(18,19,28,36)$. Because MIS staging can identify unsuspected distant metastases, it is also useful in identifying patients who need to be treated with palliative intent with treatments such as chemotherapy, esophageal stents, endoscopic laser ablation, or photodynamic therapy $(15,18)$. MIS staging may thereby help patients with metastatic esophageal cancer avoid a nontherapeutic laparotomy, which carries a mortality and morbidity rate of approximately $3 \%(27)$.

\section{Limitations of minimally invasive staging}

Some studies have questioned the need to broaden the 

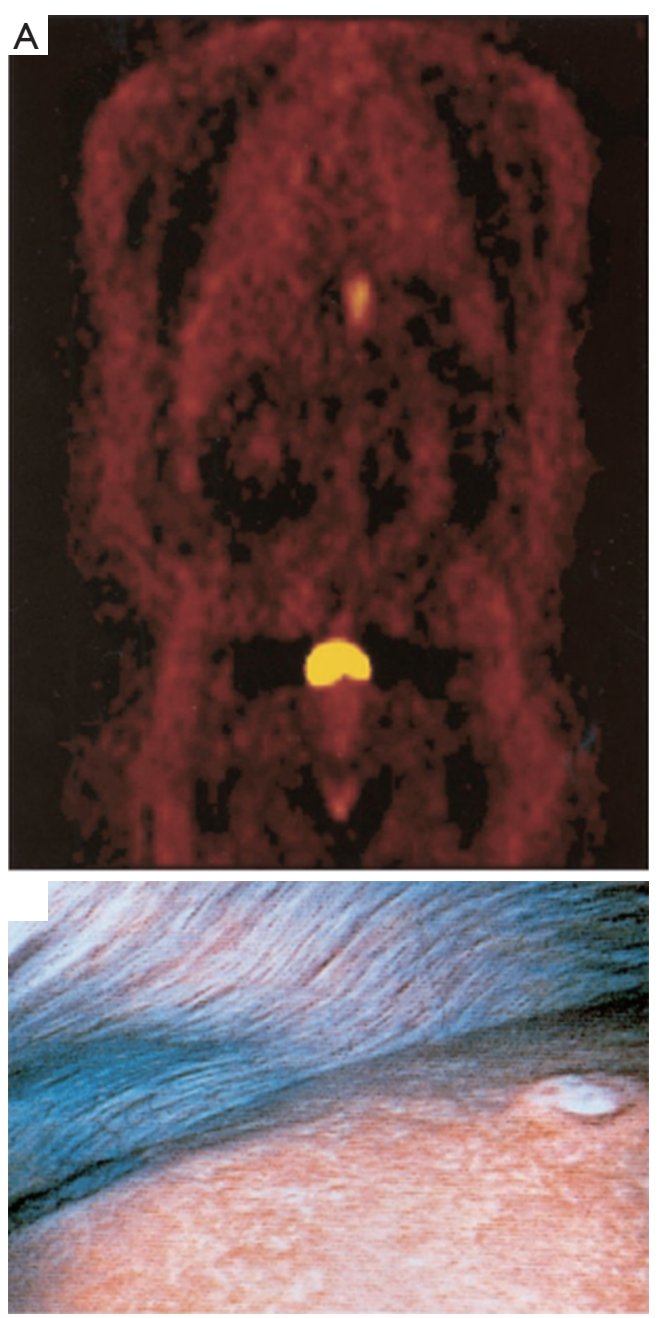

Figure 2 Laparoscopic staging detects lesions missed by PET and CT. (A) False negative PET scan demonstrating increased focal tracer uptake only at site of lesion near gastroesophageal junction; (B) in the same patient, a 6-mm liver metastatic lesion identified by laparoscopic staging, but missed by both PET and CT imaging. From reference (15). Used with permission from Elsevier.

application of minimally invasive staging in patients with esophageal cancer. Patients with distal esophageal disease, typically adenocarcinoma affecting the distal third of the esophagus or GEJ, have an increased propensity for peritoneal spread $(13,26)$. In contrast, multiple reports note that staging laparoscopy has limited utility in tumors of the upper two-thirds of the esophagus. In one study, de Graaf and colleagues noted that none of their patients with lesions affecting the proximal two-thirds of the esophagus experienced a change in management following MIS staging (26). Additional limitations of staging laparoscopy include the typical risks of intra-abdominal laparoscopy such as bleeding, infection, and injury to the esophagus or nearby structures, the cost of the procedure and the risks posed by general anesthesia.

The use of routine thoracoscopic staging in esophageal cancer also remains controversial. As previously mentioned, thoracoscopic staging of lymph nodes carries an accuracy approaching $90 \%$. The detection of metastases (e.g., to the pleura or lungs) or local invasion into unresectable structures (e.g., aorta) has been advocated as a tool to avoid an unnecessary thoracotomy in patients with unsuspected intrathoracic disease. However, no unresectable disease was identified in patients who underwent VATS staging in some studies $(11,13)$. These patients were exposed to longer operative times, and some required hospitalization (28). As such, some institutions occasionally utilize VATS staging very selectively to exclude metastases when there is concern for metastasis (e.g., to the lung), or involvement of adjacent organs such as the aorta.

\section{Conclusions}

Accurate preoperative staging of esophageal cancer is imperative to select appropriate treatments for the patients. Minimally invasive staging techniques, such as of laparoscopy and VATS (performed selectively) provide a more accurate assessment of advanced locoregional disease and distant metastases as compared with conventional imaging techniques like CT, PET, or EUS. Minimally invasive staging can be considered for patients deemed to have resectable cancers by conventional preoperative imaging to identify those who may have unidentified distant metastases, thus avoiding a nontherapeutic laparotomy, and identify unsuspected locally advanced tumors, where a multimodality strategy should be utilized. At the University of Pittsburgh Medical Center, our primary approach to esophageal resection is with a minimally invasive approach, and we perform a large number of minimally invasive esophagectomies $(37,38)$. With the shift in epidemiology and the increasing incidence of adenocarcinoma, primarily located in the distal esophagus and gastroesophageal junction, we routinely proceed with laparoscopy prior to definitive resection due to its enhanced ability to detect small foci of metastatic disease. We have found that laparoscopy is particularly valuable in detection of occult distant metastases and exclusion of patients from definitive resection. This can be accomplished prior to performing 
a definitive resection by either minimally invasive or open techniques (38). We perform staging thoracoscopy infrequently, and more selectively. It can be added very selectively in patients in whom there is concern for distant metastasis following noninvasive imaging. Further studies are required to clearly define the role of minimally invasive staging in esophageal cancer.

\section{Acknowledgements}

We thank Shannon Wyszomierski, PhD for editorial assistance during manuscript preparation.

Funding: This work was supported in part by NIH grant 5RO1 CA090665 09 and the Sampson Family Endowed Chair, University of Pittsburgh.

\section{Footnote}

Conflicts of Interest: The authors have no conflicts of interest to declare.

\section{References}

1. Pennathur A, Gibson MK, Jobe BA, et al. Oesophageal carcinoma. Lancet 2013;381:400-12.

2. What are the Key Statistics about Esophagus Cancer. cancer.org: American Cancer Society; [updated January 5, 2017; cited March 24, 2017]. Available online: http:// www.cancer.org/cancer/esophaguscancer/detailedguide/ esophagus-cancer-key-statistics

3. Enzinger PC, Mayer RJ. Esophageal cancer. N Engl J Med 2003;349:2241-52.

4. Shahbaz Sarwar CM, Luketich JD, Landreneau RJ, et al. Esophageal cancer: an update. Int J Surg 2010;8:417-22.

5. Pennathur A, Farkas A, Krasinskas AM, et al. Esophagectomy for T1 esophageal cancer: outcomes in 100 patients and implications for endoscopic therapy. Ann Thorac Surg 2009;87:1048-54; discussion 1054-5.

6. Esophagus and esophagogastric junction. In: Edge SB, American Joint Committee on Cancer., editors. AJCC cancer staging manual. 7th ed. New York: Springer; 2010:103-15.

7. Tangoku A, Yamamoto Y, Furukita Y, et al. The new era of staging as a key for an appropriate treatment for esophageal cancer. Ann Thorac Cardiovasc Surg 2012;18:190-9.

8. Berry MF. Esophageal cancer: staging system and guidelines for staging and treatment. J Thorac Dis 2014;6
Suppl 3:S289-97.

9. Rice TW, Rusch VW, Ishwaran H, et al. Cancer of the esophagus and esophagogastric junction: data-driven staging for the seventh edition of the American Joint Committee on Cancer/International Union Against Cancer Cancer Staging Manuals. Cancer 2010;116:3763-73.

10. Pennathur A, Xi L, Litle VR, et al. Gene expression profiles in esophageal adenocarcinoma predict survival after resection. J Thorac Cardiovasc Surg 2013;145:50512; discussion 512-3.

11. Krasna MJ. Minimally invasive staging for esophageal cancer. Chest 1997;112:191S-4S.

12. Karaosmanoğlu AD, Blake MA. Applications of PET-CT in patients with esophageal cancer. Diagn Interv Radiol 2012;18:171-82.

13. Luketich JD, Meehan M, Nguyen NT, et al. Minimally invasive surgical staging for esophageal cancer. Surg Endosc 2000;14:700-2.

14. Meyers BF, Downey RJ, Decker PA, et al. The utility of positron emission tomography in staging of potentially operable carcinoma of the thoracic esophagus: results of the American College of Surgeons Oncology Group Z0060 trial. J Thorac Cardiovasc Surg 2007;133:738-45.

15. Luketich JD, Friedman DM, Weigel TL, et al. Evaluation of distant metastases in esophageal cancer: 100 consecutive positron emission tomography scans. Ann Thorac Surg 1999;68:1133-6; discussion 1136-7.

16. Trindade AJ, Berzin TM. Clinical controversies in endoscopic ultrasound. Gastroenterol Rep (Oxf) 2013;1:33-41.

17. Goldfarb M, Brower S, Schwaitzberg SD. Minimally invasive surgery and cancer: controversies part 1. Surg Endosc 2010;24:304-34.

18. Luketich JD, Schauer P, Landreneau R, et al. Minimally invasive surgical staging is superior to endoscopic ultrasound in detecting lymph node metastases in esophageal cancer. J Thorac Cardiovasc Surg 1997;114:817-21; discussion 821-3.

19. Kaushik N, Khalid A, Brody D, et al. Endoscopic ultrasound compared with laparoscopy for staging esophageal cancer. Ann Thorac Surg 2007;83:2000-2.

20. Bianco V, Sablowski M, Mehta KS, Hamilton A, Odell D, Gooding WE, et al. Evaluation of the Accuracy of Endoscopic Ultrasound for Stage I Esophageal Cancer. Journal of the American College of Surgeons.219:S29.

21. Bergeron EJ, Lin J, Chang AC, et al. Endoscopic ultrasound is inadequate to determine which T1/ T2 esophageal tumors are candidates for endoluminal 
therapies. J Thorac Cardiovasc Surg 2014;147:765-71:

Discussion 771-3.

22. Huntington JT, Walker JP, Meara MP, et al. Endoscopic mucosal resection for staging and treatment of early esophageal carcinoma: a single institution experience. Surg Endosc 2015;29:2121-5.

23. Maish MS, DeMeester SR. Endoscopic mucosal resection as a staging technique to determine the depth of invasion of esophageal adenocarcinoma. Ann Thorac Surg 2004;78:1777-82.

24. Varghese TK Jr, Hofstetter WL, Rizk NP, et al. The society of thoracic surgeons guidelines on the diagnosis and staging of patients with esophageal cancer. Ann Thorac Surg 2013;96:346-56.

25. "NCCN Clinical Practice Guidelines in Oncology: Esophageal and Esophagogastric Junction Cancers." National Comprehensive Cancer Network. N.p., 2015. Web. 17 Jan. 2016.

26. de Graaf GW, Ayantunde AA, Parsons SL, et al. The role of staging laparoscopy in oesophagogastric cancers. Eur J Surg Oncol 2007;33:988-92.

27. Krasna MJ, Flowers JL, Attar S, et al. Combined thoracoscopic/laparoscopic staging of esophageal cancer. J Thorac Cardiovasc Surg 1996;111:800-6; discussion 806-7.

28. Nguyen NT, Roberts PF, Follette DM, et al. Evaluation of minimally invasive surgical staging for esophageal cancer. Am J Surg 2001;182:702-6.

29. Krasna MJ. Role of thoracoscopic lymph node staging for lung and esophageal cancer. Oncology (Williston Park)

Cite this article as: Mehta $\mathrm{K}$, Bianco V, Awais O, Luketich JD, Pennathur A. Minimally invasive staging of esophageal cancer. Ann Cardiothorac Surg 2017;6(2):110-118. doi: 10.21037/ acs.2017.03.18
1996;10:793-802; discussion 804, 813-4.

30. Krasna MJ, Reed CE, Nedzwiecki D, et al. CALGB 9380: a prospective trial of the feasibility of thoracoscopy/ laparoscopy in staging esophageal cancer. Ann Thorac Surg 2001;71:1073-9.

31. Chang L, Stefanidis D, Richardson WS, et al. The role of staging laparoscopy for intraabdominal cancers: an evidence-based review. Surg Endosc 2009;23:231-41.

32. Romijn MG, van Overhagen H, Spillenaar Bilgen EJ, et al. Laparoscopy and laparoscopic ultrasonography in staging of oesophageal and cardial carcinoma. Br J Surg 1998;85:1010-2.

33. Heath EI, Kaufman HS, Talamini MA, et al. The role of laparoscopy in preoperative staging of esophageal cancer. Surg Endosc 2000;14:495-9.

34. Hünerbein M, Rau B, Hohenberger $\mathrm{P}$, et al. The role of staging laparoscopy for multimodal therapy of gastrointestinal cancer. Surg Endosc 1998;12:921-5.

35. Molloy RG, McCourtney JS, Anderson JR. Laparoscopy in the management of patients with cancer of the gastric cardia and oesophagus. Br J Surg 1995;82:352-4.

36. Krasna MJ. Radiographic and endosonographic staging in esophageal cancer. Thorac Surg Clin 2013;23:453-60.

37. Luketich JD, Pennathur A, Awais O, et al. Outcomes after minimally invasive esophagectomy: review of over 1000 patients. Ann Surg 2012;256:95-103.

38. Pennathur A, Luketich JD. Resection for esophageal cancer: strategies for optimal management. Ann Thorac Surg 2008;85:S751-6. 